\title{
Migliorare la comunicazione in ambulatorio riduce la prescrizione degli antibiotici per le infezioni respiratorie: i risultati di un $\mathrm{RCT}$
}

Kronman MP, Gerber JS, Grundmeier RW, et al.

Reducing Antibiotic Prescribing in Primary Care for Respiratory Illness

Pediatrics. 2020;146(3):e20200038

Una comunicazione efficace con i genitori in ambulatorio permette di ridurre le prescrizioni antibiotiche inappropriate nelle cure primarie per le infezioni delle alte vie respiratorie (ARTI). In questo RCT americano con cuneo graduale, di buona qualità metodologica, il programma di formazione a distanza 'DIALOGUE AROUND RESPIRATORY ILLNESS TREATMENT' (DART QI), permette una riduzione complessiva del $7 \%$ della probabilità di prescrizione di antibiotici per ARTI tra il periodo basale e post-intervento.

Improving communication in the office reduces the prescription of antibiotics for respiratory infections: the results of an RCT Effective communication with parents in the clinic can reduce inappropriate antibiotic prescriptions in primary care for upper respiratory tract infections (URTI). In this American RCT with a gradual wedge of good methodological quality, the distance training program 'DIALOGUE AROUND RESPIRATORY ILLNESS TREATMENT' (DART QI), allows an overall reduction of $7 \%$ in the probability of prescribing antibiotics for URTI between the baseline period and post-surgery.

\section{Metodo}

\section{Obiettivo (con tipo studio)}

RCT con cuneo graduale (Stepped Wedge Clinical Trial (Glossario) con l'obiettivo di valutare l'efficacia di un programma di insegnamento a distanza DART QI, nella riduzione della prescrizione di antibiotici durante le visite per infezioni respiratorie.

\section{Popolazione}

19 poliambulatori pediatrici con 57 pediatri (da 1 a 6 pediatri per studio) in 9 stati americani appartenenti a due gruppi di ricerca pediatrica ambulatoriale (AAP Pediatric Research in Office Settings; North Shore University Health System) assegnati in modo casuale a 4 gruppi che hanno ricevuto in tempi diversi i 3 moduli dell'intervento per migliorare la prescrizione degli antibiotici nelle infezioni delle vie respiratorie.

\section{Intervento}

I medici hanno ricevuto l'intervento DART QI attraverso 3 moduli di programma contenenti:

- tutorial online e webinar su strategie di comunicazione eviden- ce based e indicazioni per la prescrizione di antibiotici; - video con vignette di richiamo;

- rapporti di feedback sulla prescrizione di antibiotici individualizzati nell'arco di 11 mesi.

\section{Controllo}

Il materiale è disponibile online, le strategie comunicative proposte sono sintetizzate nel Box 1.

Il gruppo di controllo è stato rappresentato dagli stessi medici arruolati e randomizzati e il confronto è stato fatto prima, durante i diversi moduli e al termine dell'intervento per tutti i gruppi che lo hanno ricevuto (Glossario).

\section{Outcome/Esiti}

Outcome primario: totale dei tassi di prescrizione di antibiotici per tutte le visite per ARTI (otite media acuta, bronchite, faringite, sinusite e infezione delle vie respiratorie superiori per i bambini da 6 mesi a 11 anni senza l'uso recente di antibiotici nell' ultimo mese e senza diagnosi associata di patologia batterica). Outcome secondario:

1. tassi di prescrizione per visita per ARTI virali;

\section{Glossario}

\section{Stepped Wedge Clinical Trial}

Uno studio a cuneo graduale (o Stepped Wedge Clinical Trial - SWT) è un tipo di studio randomizzato controllato (o RCT strutturato) per ridurre i bias quando si testano nuovi trattamenti o interventi. In un RCT tradizionale, una parte dei partecipanti all'esperimento viene assegnata simultaneamente e casualmente a un gruppo che riceve il trattamento (il "gruppo di trattamento") e un'altra parte a un gruppo che non lo fa (il "gruppo di controllo"). In un SWT tutti o la maggior parte dei partecipanti ricevono il trattamento con una diversa modularità. Lo SWT comporta la raccolta di osservazioni durante un periodo di riferimento in cui nessun gruppo è esposto all'intervento. Successivamente, a intervalli regolari, o moduli, un gruppo o una serie di gruppi viene randomizzato per ricevere l'intervento e tutti i partecipanti vengono nuovamente misurati. Questo processo continua fino a quando tutti i gruppi non hanno ricevuto l'intervento. Infine, un'altra misurazione viene effettuata dopo che tutti i gruppi hanno ricevuto l'intervento. Questo tipo di disegno è particolarmente utile negli studi pragmatici. Inoltre, di solito gli SWT richiedono una dimensione del campione più piccola per rilevare gli effetti poiché sfruttano i confronti sia tra che all'interno del gruppo. 
2. tassi di prescrizione per visita per faringite;

3. prescrizione di antibiotici di seconda linea per otite media acuta AOM;

4. prescrizione di antibiotici di seconda linea per faringite da streptococco;

5. prescrizione di antibiotici di seconda linea per sinusite.

\section{Tempo}

Da novembre 2015 a giugno 2018. L'intervento è iniziato in momenti diversi nei 4 gruppi tra gennaio e giugno 2017 e si è concluso 11 mesi dopo; il periodo di controllo di base considerato è stato da novembre 2015 all'inizio dell'intervento nel 2017; il periodo post-intervento considerato è stato a seconda del gruppo di randomizzazione di 2-8 mesi (novembre 2017- giugno 2018).

\section{Risultati principali}

Nelle 72.723 visite ARTI valutate nel periodo di studio riguardanti 29.762 pazienti, il 39.5\% delle quali associata a una prescrizione antibiotica, è stata rilevata una diminuzione del 7\% della probabilità di prescrizione di antibiotici per ARTI complessiva tra il periodo basale e post-intervento (aRR 0.93; IC 95\% 0.90, 0.96). Il massimo effetto dell' intervento è stato raggiunto durante il secondo modulo con una riduzione delle prescrizioni del $16 \%$
(IC 95\% 12\%, 19\%). Qualsiasi prescrizione di antibiotici è diminuita nelle ARTI virali (aRR 0.60; IC 95\% 0.51, 0.70). La prescrizione di antibiotici di seconda linea è diminuita per la faringite streptococcica (aRR 0.66; IC 95\% 0.50, 0.87) e la sinusite (aRR 0.59 ; IC 95\% 0.44, 0.77) ma non per l'otite media acuta (aRR 0.93 ; IC 95\% 0.83, 1.03).

\section{Conclusioni}

Il programma di insegnamento a distanza DART QI è efficace nel ridurre la prescrizione di antibiotici durante le visite per ARTI e la sua diffusione potrebbe avere effetti benefici consentendo una diminuzione significativa di prescrizioni antibiotiche improprie. Gli autori stimano che a livello nazionale, negli USA, questo intervento comporterebbe una riduzione annuale di 1.5 milioni di prescrizioni antibiotiche pediatriche per ARTI.

\section{Altri studi sull'argomento}

Una revisione sistematica del 2014 degli studi che hanno valutato la comunicazione tra genitore e pediatra durante visite per malattie acute ha incluso 13 studi, 7 su ARTI e 6 su bambini con qualsiasi malattia. L'analisi ha rilevato che la comunicazione del genitore si è concentrata sulle sue preoccupazioni per il bambino

\section{Box 1}

Strategie comunicative efficaci per ridurre la prescrizione antibiotica per ARTI

[modificato da: DART (DIALOGUE AROUND RESPIRATORY ILLNESS TREATMENT) https://www.uwimtr.org/dart/ ]

Il modo di comunicare dei genitori influenza fortemente le prescrizioni antibiotiche: se il medico percepisce che il genitore vuole l'antibiotico è più probabile che lo prescriva anche se non necessario. Utilizzare strategie di comunicazione efficace permette di evitare questo.

\section{Come riconoscere l'aspettativa genitoriale di prescrizione antibiotica in una visita ambulatoriale per ARTI}

L'aspettativa genitoriale di una prescrizione antibiotica influenza il modo di comunicare dei genitori durante la visita.

È importante osservare come avviene la presentazione del problema sanitario del figlio. L'aspettativa genitoriale può essere: - esplicita: quando il genitore nomina una possibile diagnosi (ad es bronchite).

- implicita: quando il genitore descrive i sintomi in un modo che implica una diagnosi specifica (ad es. scolo nasale mucopurulento verdastro) oppure afferma che qualcun altro in famiglia o a scuola ha ad esempio la faringite streptococcica o la sinusite.

\section{Come gestire in modo efficace l'aspettativa genitoriale di una prescrizione antibiotica}

Ci sono tecniche di comunicazione che possono avere successo in queste aspettative:

1. Parlare con il genitore per rivedere ciò che si è trovato alla visita medica del bambino, ad es. "lobiettività cardio-polmonare è nella norma, le orecchie non presentano segni di otite, la gola è arrossata ma non ci sono placche, il naso è ostruito e cè secrezione mucosa".

2. Riferire al genitore una diagnosi certa, ad es. "Suo figlio ha il raffreddore".

3. Strutturare le raccomandazioni terapeutiche partendo da una raccomandazione negativa che escluda l'antibiotico, seguita da una prescrizione positiva per ridurre i sintomi, ad es. "Per il raffreddore l'antibiotico non serve perché non aiuterebbe il bambino a migliorare (raccomandazione negativa alla terapia); ci sono, però, molte cose che si possono fare per alleviare i sintomi come alzare il capo con un cuscino mentre il bambino dorme per migliorare lo scolo nasale e diminuire la tosse notturna e offrire spesso acqua da bere per fluidificare il muco (raccomandazione positiva)". I genitori infatti si sentono frustrati quando il medico dice che non è necessaria alcuna terapia (ad es. "È un raffreddore, non serve una terapia, guarirà spontaneamente") poiché la loro aspettativa è di ricevere informazioni su come trattare i sintomi del bambino per farlo stare meglio; quindi è importante che il medico non abbia un atteggiamento comunicativo che tende a minimizzare il problema. Negare una terapia aumenta la richiesta di un piano terapeutico; se il medico rimanda la decisione su cosa fare inizia una negoziazione impropria medico/genitore. Questa situazione aumenta la probabilità di una prescrizione impropria e, inoltre, aumenta la durata della visita forzando il medico a rispiegare perché l'antibiotico non è necessario. Quando il medico combina la prescrizione che nega l'antibiotico seguita da una raccomandazione positiva che riduca i sintomi si ottiene una bassa incidenza di prescrizioni improprie, ed è presente una associazione con un'alta soddisfazione genitoriale e una riduzione dei tempi di visita.

4. Fornire al genitore un piano terapeutico contingente, cioè consigliare al genitore che se nei giorni successivi il bambino non migliorasse può ricontattare il medico, per telefono o ritornando in ambulatorio e la terapia potrebbe cambiare. La disponibilità del medico a una rivalutazione aumenta significativamente il grado di soddisfazione del genitore. 
e sulla necessità di informazione, mentre la comunicazione del medico si è concentrata sulla diagnosi e sul trattamento. Durante gli scambi di informazioni, i genitori spesso cercano di giustificare la necessità della visita, mentre i medici usano un linguaggio che tende a minimizzare il problema, si genera quindi un mismatch comunicativo. Nel contesto delle ARTI, una serie di comportamenti comunicativi dei genitori sono stati interpretati dai medici come indicanti un'aspettativa di prescrizione antibiotica, tuttavia, la maggior parte erano ambigui e potevano anche essere interpretati come preoccupazione o richiesta di ulteriori informazioni. La percezione dell'aspettativa per gli antibiotici spesso ha cambiato il processo decisionale clinico nella negoziazione medico-genitore. Gli autori concludono che ai medici delle cure primarie dovrebbe essere offerta una formazione sulla comunicazione [1]. Uno studio inglese del 2016 degli stessi autori ha analizzato le dinamiche comunicative nell'ambulatorio del pediatra durante una visita per ARTI. Il modo in cui i medici di solito comunicano una diagnosi di ARTI virale avviene attraverso un linguaggio che tende a minimizzare il problema percepito dai genitori. Anche se il pediatra informa il genitore che gli antibiotici non sono efficaci contro i virus, se non offre una risposta concreta al problema portato dal genitore, ad esempio indicando una terapia di supporto per il controllo dei sintomi di ARTI, il genitore non modifica le sue convinzioni in materia di antibiotici e necessità di consultazione del medico. Spesso la modalità di comunicazione del medico porta il genitore a convincersi che gli antibiotici sono necessari per malattie più gravi e quindi a richiederne la prescrizione anche implicitamente per paura che si generi nel figlio un problema di salute importante. I genitori capiscono poco i concetti legati all'antibiotico-resistenza mentre comprendono meglio la necessità di ridurre prescrizioni inutili, anche se alcuni credono che la necessità di ridurre le prescrizioni antibiotiche dipenda dal fatto che c'è un razionamento delle risorse e non per l'inutilità e l'eventuale danno di una terapia antibiotica inappropriata [2]. Uno studio condotto nella regione Emilia-Romagna nel 2009 ha evidenziato che, nelle visite per ARTI, sebbene i pediatri non ritengano importante l'aspettativa dei genitori rispetto alla prescrizione antibiotica, questo è un determinante cruciale della prescrizione, risultando il secondo fattore più fortemente associato $(\mathrm{OR}=12.8$; $\mathrm{IC}$ $95 \% 10.4,15.8)$ dopo l'otorrea. Per quanto riguarda i clinici l' incertezza diagnostica è ciò che viene percepito essere il fattore più importante che porta a prescrivere un antibiotico nelle ARTI, mentre per quanto riguarda i genitori i più importanti fattori identificati, potenzialmente associati alla sovraprescrizione, sono la mancanza di conoscenza delle ARTI e degli antibiotici (il $41 \%$ dei 1029 genitori ha indicato i batteri come un possibile causa del comune raffreddore), e la propensione a cercare cure mediche per infezioni banali (48\% di 4.352 bambini visitati nell'ambulatorio del pediatra, presentavano solo sintomi di raffreddore comune). L'ampio divario che esiste tra determinanti percepiti e reali della prescrizione di antibiotici può promuovere l'uso eccessivo di antibiotici [3].

\section{Che cosa aggiunge questo studio}

Questo studio dimostra che anche in ambito pediatrico, la combinazione di un intervento che associa formazione online sulle strategie di comunicazione insieme alla formazione per la pre- scrizione antibiotica appropriata, basate su prove di efficacia, insieme ai report individualizzati sulle prescrizioni effettuate dai singoli clinici è efficace per ridurre le prescrizioni antibiotiche per ARTI nei bambini.

\section{Commento}

\section{Validità interna}

Disegno dello studio: lo studio è uno studio pragmatico a cuneo graduale a coorte chiusa e presenta buona qualità metodologica. È stata discussa la potenza dello studio. Sono state utilizzate l'ITT e l'analisi multivariata che ha tenuto conto di variabili precedentemente associate a un maggior impiego degli antibiotici. E stata fatta una analisi per sottogruppi distinguendo chi aveva completato tutto l'intervento rispetto a chi ne aveva effettuato solo una parte. Gli autori segnalano che due medici hanno lavorato in gruppi randomizzati in blocchi diversi ma poiché l'intervento è individuale questo non dovrebbe aver alterato significativamente l'esito dello studio. Inoltre è stato monitorato un possibile effetto Hawthorne (Glossario 2). Il fatto che non siano state registrate particolari variazioni nel numero di ARTI batteriche registrate prima e dopo l'intervento suggerisce che i medici non hanno modificato la diagnosi per giustificare una eventuale terapia antibiotica. Il periodo di follow-up considerato al termine dell'intervento di 2-8 mesi non è breve ma occorrerebbe verificare in tempi più lunghi se l'effetto si mantiene o se può essere utile un rinforzo a distanza.

Esiti: rilevanti e ben definiti.

Conflitto di interesse: nessuno.

\section{Trasferibilità}

Popolazione studiata: è sovrapponibile a quella che accede nei nostri ambulatori. Come in USA anche in Italia è presente un' iperprescrizione di antibiotici. Il rapporto OSMED (AIFA) sull'uso dei farmaci in Italia nel 2020 conferma che, nonostante la riduzione registrata nel 2020 a causa della bassa incidenza di infezioni respiratorie favorita dal periodo di lock-down, gli antinfettivi per uso sistemico si confermano la categoria terapeutica a maggiore prevalenza prescrittiva in età pediatrica (475.6 per 1.000 bambini nel 2020 contro 1.013/1.000 bambini del 2019), con l'associazione amoxicillina/acido clavulanico che risulta comunque essere il farmaco più prescritto della categoria (183 prescrizioni per 1.000 bambini).

Tipo di intervento: potrebbe essere utile e applicabile nel nostro contesto. La fruibilità individuale in asincrono online da parte di ciascun clinico e il fatto che è una formazione breve (alcune ore di formazione in tempi frazionati) rappresentano un punto di forza dell'intervento. Attualmente, per nostra conoscenza, nel nostro Paese non sono stati attuati interventi di formazione sulla strategia comunicativa con l'obiettivo di ridurre la prescrizione antibiotica. Alcune regioni italiane come l'Emilia-Romagna e il Piemonte hanno attuato con beneficio percorsi di sorveglianza dell'appropriatezza prescrittiva che hanno permesso di ridurre nel tempo le prescrizioni antibiotiche. In Piemonte il tasso di prescrizione di antibiotici è passato da 931 a 689 ogni mille bambini dal 2012 al 2018, con un 34\% complessivo di bambini in terapia, con amoxicillina + acido clavulanico come molecola più prescritta (420 prescrizioni/1.000 bambini nel 2018) [4]. In Emi- 
lia-Romagna le prescrizioni antibiotiche si sono ridotte dal 2005 al 2017 e successivamente sono nuovamente lievemente aumentate nel biennio 2018-2019, per poi dimezzarsi nel 2020. La riduzione complessiva dal 2005 al 2019 è stata del 37\%: da 1.307 a 822 ogni 1.000 bambini (402/1.000 nel 2020), con un 41\% di bambini nel 2019 che hanno ricevuto almeno un antibiotico $[5,6]$, valori quindi ancora lontani da quelli desiderabili di alcuni paesi europei come i Paesi Bassi in cui solo il 15\% della popolazione pediatrica riceve annualmente almeno una prescrizione antibiotica, con un rapporto amoxicillina/amoxicillina + ac. clavulanico superiore a 4 [7]. In Emilia-Romagna nel 2019 la molecola più prescritta è risultata essere l'amoxicillina, con un rapporto prescrittivo amoxicillina/amoxicillina + ac. clavulanico di 1.5 che è leggermente peggiorato nel 2020 diventando di 1.3. Una formazione sulle strategie comunicative da utilizzare in ambulatorio potrebbe integrarsi ai feedback sulle prescrizioni individuali per migliorare ulteriormente l'appropriatezza prescrittiva riducendo prescrizioni antibiotiche per ARTI virali.

1. Cabral C, Horwood J, Hay AD et al. How communication affects prescription decisions in consultations for acute illness in children: a systematic review and meta-ethnography. BMC Fam Pract. 2014; 15: 63. 2. Cabral C, Ingram J, Lucas PJ et al. Influence of Clinical Communication on Parents' Antibiotic Expectations for Children With Respiratory Tract Infections. Ann Fam Med. 2016 Mar; 14(2): 141-147

3. Moro ML, Marchi M, Gagliotti C, et al. the "Progetto Bambini a Antibiotici [ProBA]" Regional Group Why do paediatricians prescribe antibiotics? Results of an Italian regional project BMC Pediatr. 2009; 9: 69. 4. Ravaglia A, Guala A, Gnavi R, et al. Prescrizione di antibiotici in Pediatria tra il 2012 e il 2018 in Regione Piemonte. Medico e Bambino 2019; 38:575-580.

5. Gagliotti C, Buttazzi R, Ricchizzi E, et al. Uso di antibiotici e resistenze antimicrobiche in età pediatrica. Rapporto Emilia-Romagna 2019 Agenzia sanitaria e - sociale regionale (regione.emilia-romagna.it)

6. Gagliotti C, Buttazzi R, Ricchizzi E, et al. Uso di antibiotici e resistenze antimicrobiche in età pediatrica. Rapporto Emilia-Romagna 2020 Agenzia sanitaria e -sociale regionale (regione.emilia-romagna.it)

7. Clavenna A, Bonati M. Differences in antibiotic prescribing in paediatric outpatients. Arch Dis Child 2011;96(6):590-5.

\section{Scheda redatta dal gruppo di lettura di Parma:}

Maria Teresa Bersini, Francesca Manusia, Maddalena Marchesi, Sandra Mari, Alessandra Massari, Manuela Musetti, Luisa Mustaccio, Costantino Panza.

\section{Glossario 2}

\section{Effetto Hawthorne}

L'effetto Hawthorne è il miglioramento dei risultati che si determina quando si entra a far parte di una ricerca ma che non è duraturo nel tempo. Per evitare che questo confonda i risultati può essere utile che anche il gruppo di controllo riceva un minimo intervento e non soltanto sia osservato. 